\title{
Classifications in Brief: The Wassel Classification for Radial Polydactyly
}

\author{
Mary Claire Manske MD, Colin D. Kennedy MD, Jerry I. Huang MD
}

Received: 7 June 2016/ Accepted: 29 August 2016/Published online: 9 September 2016

(C) The Association of Bone and Joint Surgeons \& 2016

\section{History}

Radial (preaxial) polydactyly is among the most common congenital anomalies of the hand, with an incidence of 0.08 to 1.4 per 1000 live births $[1,11,17,21]$. Although radial polydactyly is commonly described as "thumb duplication," it is uncommon that the two thumbs are truly duplicated with equal size and function. More commonly, one "dominant" thumb is more developed anatomically and functionally than the other, leading some surgeons to prefer the term "split thumb" to communicate the concept that neither thumb is fully formed nor complete. Radial polydactyly originally was classified as a "duplication" by the International Federation of Societies for the Surgery of the Hand rather than as a "failure of formation" or "failure of differentiation" [19]. It since has been reclassified as a "malformation," a failure of axis formation, and/or differentiation of the radioulnar hand plate in the Oberg, Manske, and Tonkin classification, which classifies

Each author certifies that he or she, or a member of his or her immediate family, has no funding or commercial associations (eg, consultancies, stock ownership, equity interest, patent/licensing arrangements, etc) that might pose a conflict of interest in connection with the submitted article.

All ICMJE Conflict of Interest Forms for authors and Clinical Orthopaedics and Related Research ${ }^{\circledR}$ editors and board members are on file with the publication and can be viewed on request.

Each author certifies that his or her institution approved the human protocol for this investigation, that all investigations were conducted in conformity with ethical principles of research, and that informed consent for participation in the study was obtained.

M. C. Manske ( $₫)$, C. D. Kennedy, J. I. Huang

Department of Orthopaedics and Sports Medicine, University of Washington, 4245 Roosevelt Way NE, Box 354740, Seattle, WA 98105, USA

e-mail: mclairemanske@gmail.com anomalies based on developmental biology and pathogenesis rather than on morphologic features, and recognizes that processes such as formation and differentiation occur together and not independently [15]. From the genetic perspective, most radial polydactyly occur owing to a sporadic genetic mutation, but triphalangeal thumbs are inherited in an autosomal dominant manner [26].

Despite ancient descriptions of polydactyly, the first attempts to classify thumb polydactyly were not developed until the 20th century [16]. Egawa [7] in Japan and Millesi [13] in Austria organized the various presentations of thumb polydactyly into classification systems in their native languages in 1966 and 1967, respectively. It was not until 1969 when Harry Wassel, as a hand surgery fellow of Adrian Flatt at the University of Iowa, authored "The Results of Surgery for Polydactyly of the Thumb: A Review", [24] that a classification system for radial polydactyly was published in the English scientific literature. Wassel's description of seven types of thumb polydactyly based on the level of skeletal duplication subsequently gained wide acceptance given its simplicity, clarity, and ease of use. Since its original description, many, including the membership of the Pediatric Hand Study Group (an international academic society of pediatric hand surgeons and hand therapists) call this classification system the "Flatt Classification" to acknowledge the intellectual genesis of this classification from Adrian Flatt, MD [25]. The term Flatt Classification has now been adopted in the pediatric hand surgery literature $[6,9,12,18,23,25,27]$.

\section{Purpose}

An ideal classification system is reproducible, facilitates clear documentation and communication, encompasses all 
Table 1. Wassel's recommendations for operative technique and surgical timing

\begin{tabular}{|c|c|c|}
\hline Thumb type & Operative technique & Timing \\
\hline \multirow[t]{2}{*}{ Types I, III, V } & Bilhaut-Cloquet technique if angular deformity is present & Surgery at 3 years old or older \\
\hline & $\begin{array}{l}\text { Leave bifid element intact to prevent angular deformity; } \\
\text { bifid element may be removed at skeletal maturity }\end{array}$ & $\begin{array}{l}\text { Earlier surgery has increased risk } \\
\text { of physeal damage }\end{array}$ \\
\hline Types II, V & $\begin{array}{l}\text { Ablation of supernumerary thumb with collateral ligament } \\
\text { reconstruction }\end{array}$ & Early surgical ablation \\
\hline \multirow[t]{2}{*}{ Type VII } & Ablation of triphalangeal thumb & Timing not discussed \\
\hline & Retain biphalangeal thumb even if rudimentary & \\
\hline
\end{tabular}

pathologic types of deformity, guides treatment, correlates with prognosis, and is applicable to research. The purpose of Wassel's classification system was to identify subtypes of radial polydactyly and evaluate the outcome of surgical ablation of the supernumerary (usually radial) digit, defined as "freedom from impairment of function and restoration of acceptable cosmesis" [24]. Outcome parameters included thumb opposition, interphalangeal and metacarpal phalangeal joint motion, tip pinch strength, and angular deformity of the digit. Based on these results, Wassel's manuscript provided recommendations regarding operative technique and timing of surgery for each type (Table 1).

\section{Description}

Wassel's classification system of thumb polydactyly is a radiographic description based on the level of skeletal duplication. From distal to proximal, the phalanges and metacarpal of the thumb are identified as bifid (a cleft in the bone distally with a shared base proximally), duplicated (extension of the cleft from distal to proximal, resulting in completely independent osseous structures), or unaffected. A Roman numeral is assigned based on how proximally the bifurcation or duplication extends, with odd numbers representing bifid osseous structures, even numbers indicating duplicated bones, and greater numbers describing more-proximal levels of duplication. Wassel Types I through VI are assigned according to this algorithm, but Type VII designates a triphalangeal thumb (Fig. 1). In the description of each subtype, Wassel described the potential for divergence or convergence of the duplicated digits and acknowledged the importance of angular deformity on operative technique and outcome, but did not assign classification or subclassification types based on the presence or magnitude of angular deformity.

\section{Validation}

Despite the near universal adoption of the Wassel's classification system, there have been few attempts to validate it and none published until more than 40 years after the Wassel classification was introduced.

Dijkman et al. [5] assessed the reliability of the Wassel classification system in 2014 by evaluating the intra- and interobserver agreement of seven reviewers (four congenital hand surgeons, a resident, a $\mathrm{PhD}$ candidate, and a medical student), who analyzed the radiographs of 40 cases of thumb polydactyly randomly selected from the authors' series of 520 patients with radial polydactyly. The evaluators classified each patient according to the Wassel classification at two time points 2 weeks apart. The intraobserver reliability of the Wassel classification system showed near-perfect agreement $(\mathrm{kappa}=0.87)$, although the interobserver reliability had only substantial agreement (kappa $=0.65$ ). Type-specific reliability was lowest for Wassel Types II and IV thumbs (intraobserver reliability kappa $=0.40$ and 0.50 and interobserver reliability of kappa $=0.33$ and 0.34 , respectively), indicating only moderate to fair agreement for these subtypes of thumb polydactyly. In addition, the authors evaluated the relationship between reliability of the classification system and years of experience in congenital hand surgery. Notably, there was no relationship between the experience of the evaluator and the reliability of classifying according to the Wassel system.

Cabrera Gonzalez et al. [3] evaluated the prognostic value of the Wassel classification as modified by Egawa [7] (which described Type VII thumb polydactyly as a "floating" thumb rather than a triphalangeal thumb) in predicting complications and functional outcome (Tada score [20]) after thumb reconstruction for radial polydactyly. The authors found a higher complication rate with surgical treatment of Wassel Types III and IV deformities, but not a relationship between functional outcome and Wassel type. The most common complications identified were axial angulation deformity followed by joint instability.

\section{Limitations}

Since the publication of the Wassel classification system, numerous authors have identified deficiencies [2, 4, 5, 
Fig. 1 The Wassel classification system is a radiographic description based on the level of skeletal duplication. From distal to proximal, the phalanges and metacarpal of the thumb are identified as bifid, duplicated, or unaffected, with a Roman numeral assigned based on how proximally the bifurcation or duplication extends. (Published with permission from Wassel HD. The results of surgery for polydactyly of the thumb. Clin Orthop Relat Res. 1969;64:175193.).
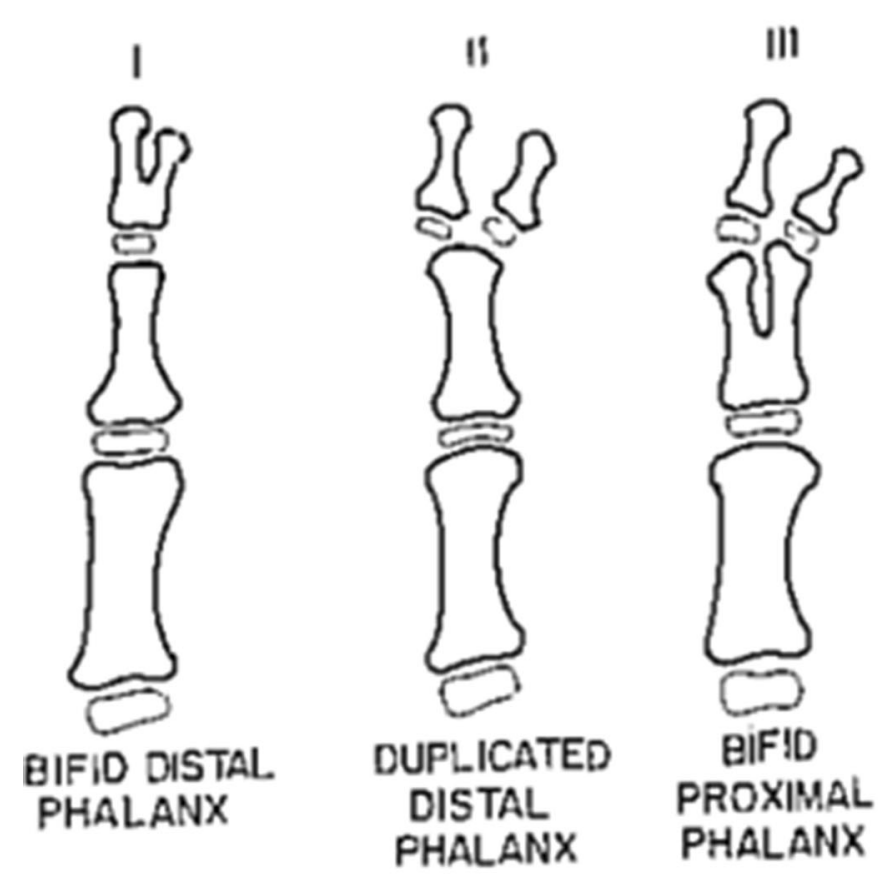

IV
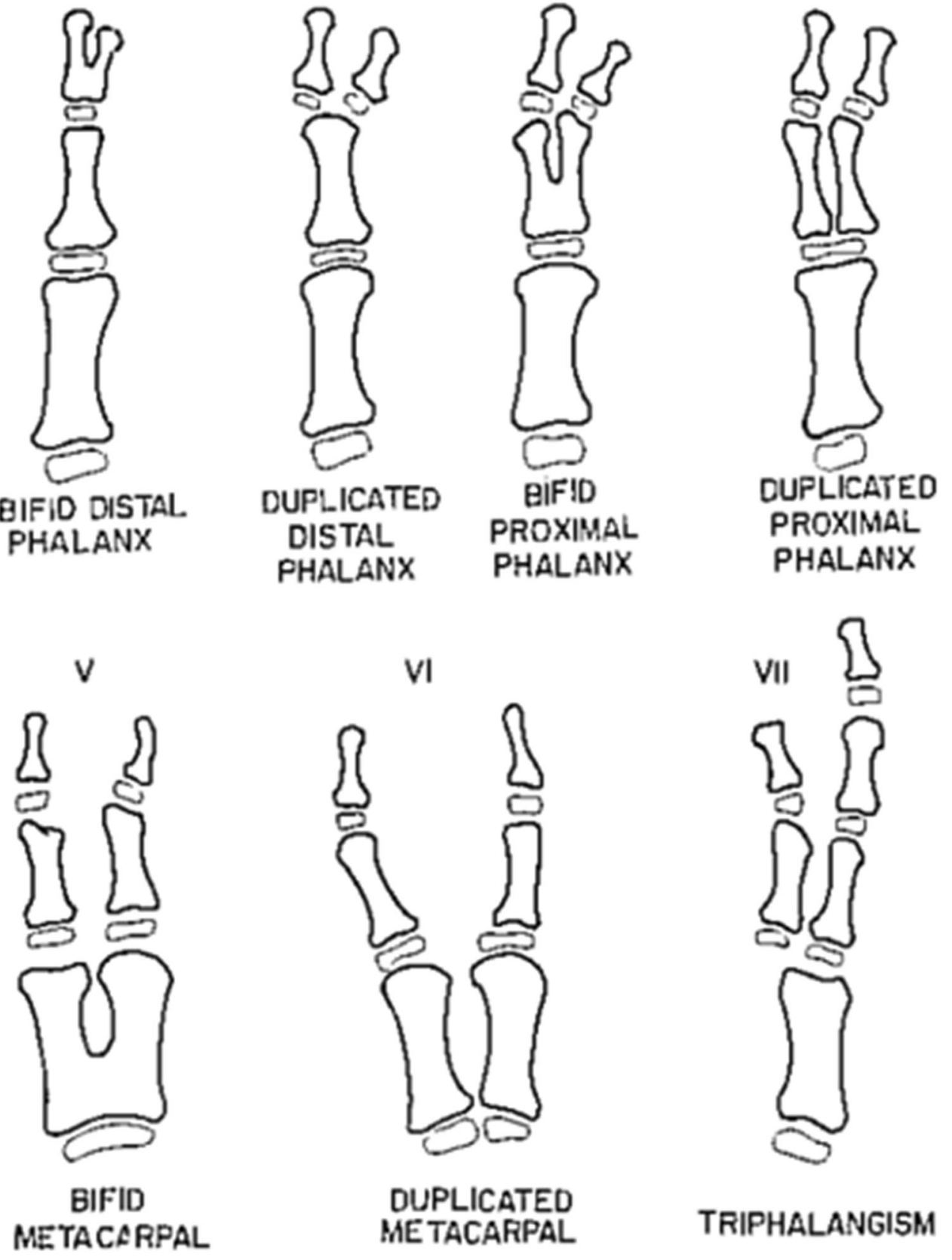

TRIPHALANGISM
$8,14,22,28,29]$, including concerns that it is not inclusive of all presentations of radial polydactyly, is insufficient to guide surgical intervention, and may not be accurate in skeletally immature patients.

Perhaps the greatest limitation of the Wassel classification is that it does not account for the spectrum of anatomic complexity of radial polydactyly and therefore is limited in its ability to classify all presentations of polydactylous thumbs. The inclusion of the triphalangeal thumb, in particular, has always been controversial. In fact,
Flatt's 1977 modification of the Wassel classification system included only Types I through VI and excluded the triphalangeal thumb, considering this presentation a distinct type of thumb deformity [10]. Similarly, BuckGramko and Behrens [2] modified Wassel's classification to include bifid trapezium and fully duplicated trapezium as Types VII and VIII, respectively, and considered triphalangism separately. Wood [28] and Miura [14] also presented modifications of Wassel's system to better accommodate triphalangeal thumbs. According to Wood's 
modification, Type IV thumbs (duplicated proximal and distal phalanges) are subdivided into three subtypes: Type A (in which both duplicated elements at the proximal phalanx level contain triphalangeal elements) and Type B (only the radial-sided duplication is triphalangeal) [28]; Miura later added Type C (only the ulnar-sided duplication thumb is triphalangeal) [14]. Wood's modification also subclassified Wassel's Type VII triphalangeal thumb into four types: Type A (a duplicated thumb at the level of the metacarpal with a triphalangeal ulnar thumb), Type B (a duplicated thumb at the level of the metacarpal in which both duplicated thumbs are triphalangeal), Type $\mathrm{C}$ (a duplicated thumb at the level of the metacarpal with a triphalangeal radial thumb), and Type D (a triplicated thumb in which the central component is triphalangeal).

To provide a comprehensive classification system inclusive of all the anatomic variations of radial polydactyly, Zuidam et al. [29] proposed the Rotterdam classification system in 2008, which combines the basic scheme of Wassel's classification system and Buck-
Gramko and Behren's intercarpal modification and introduces suffixes to indicate different complex deformities such as triphalanagism and triplication (Fig. 2). According to this classification system, which uses a similar scheme as Wassel's description (proceeding from distal to proximal, odd numbers for bifurcation and even numbers for duplication), the level of duplication is represented by a Roman numeral with Type I representing a bifid distal phalanx and Type VIII representing a duplicated trapezium with odd numbers representing bifid structures and even numbers representing complete duplications. Associated deformities are designated with the following suffixes: Tph (triphlangism), T (triplication), S (symphalangism), D (duplication), and $\mathrm{H}$ (hypoplasia). Finally the position of these complex deformities is indicated by $\mathrm{r}$ (radial), $\mathrm{m}$ (middle), and $\mathrm{u}$ (ulnar).

Dijkman et al. [5] evaluated the ability of the Wassel system to classify the various presentations of thumb polydactyly and found that 208 of their series of 520 duplicated thumbs (40\%) could not be classified using the

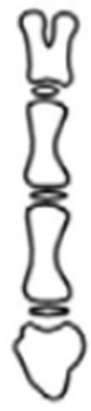

I

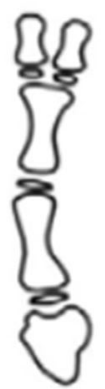

II

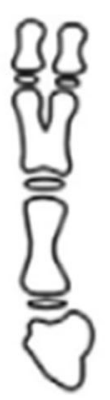

III

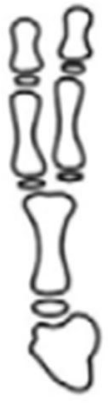

IV

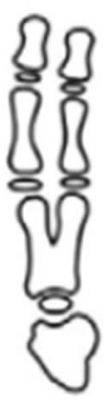

V

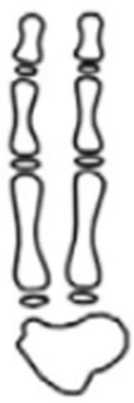

VI

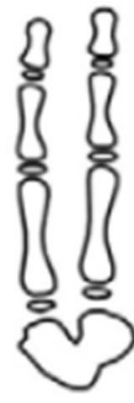

VII

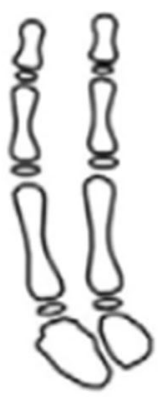

VIII

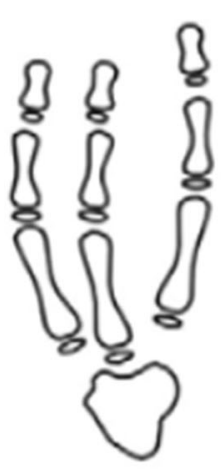

Triplication

T

Type VI T

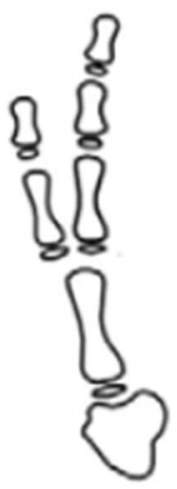

Triphalangism Tph

Type IV Tph u
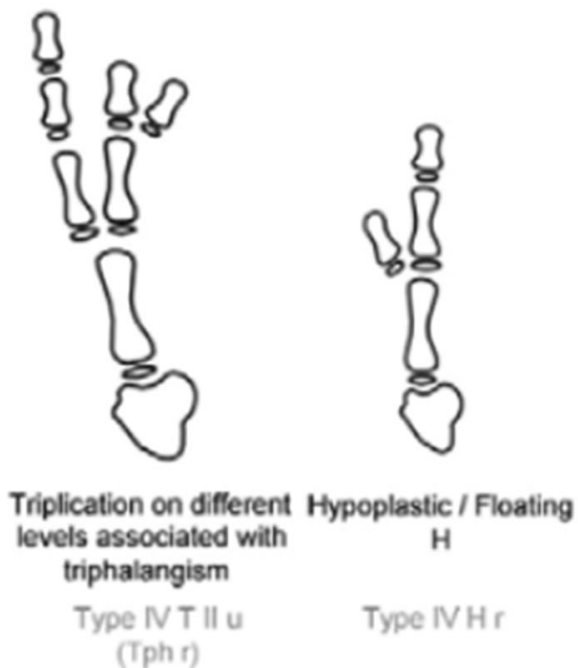
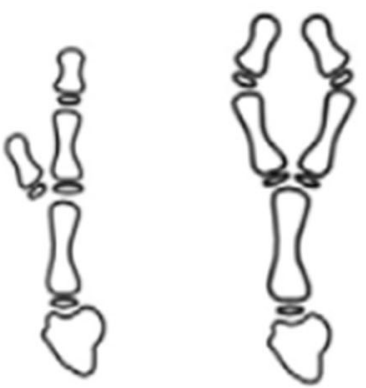

Deviation

D

Type IV D u'r

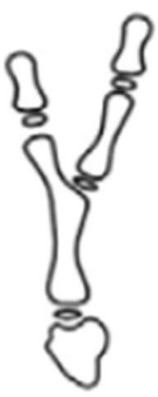

Symphalangism S
Fig. 2 The Rotterdam classification of radial polydactyly as described by Zuidam et al. [29] is shown. (Published with permission from Zuidam JM, Selles RW, Ananta M, Runia J, Hovius SE. A classification system of radial polydactyly: inclusion of triphalangeal thumb and triplication. J Hand Surg Am. 2008;33:373-377.). 
Wassel system. In addition, they reviewed 1723 cases of radial polydactyly in the medical literature and were unable to classify 96 of 1723 cases (6\%) using the Wassel system. The unclassifiable cases were most commonly triphalangeal $(63 \%)$, deviating (43\%), or hypoplastic (39\%). Using the Rotterdam classification scheme, they were able to classify all presentations of thumbs in their series of 520 cases.

The second major critique of the Wassel classification system is its limited ability to guide surgical intervention of the polydactylous thumb because certain characteristics that influence surgical treatment are not included. In particular, the Wassel classification does not provide insight into which thumb is more developed or functionally dominant, the presence of angular deformity (convergence or divergence of the digits), joint instability, irregularities of the articular surface (namely, broad or bifid joints), or additional soft tissue anomalies (including ligamentous deficiencies or aberrant tendon insertions) [17] (Fig. 3). These characteristics are critical to recognize as they inform operative decision-making and influence surgical outcomes. In particular, thumb duplications with convergence and divergence (the so-called "zigzag duplication") result from abnormal joint alignment, eccentric tendon insertions, and collateral ligament insufficiency. Careful surgical technique is required to address all elements contributing to the deformity, optimize the appearance and function of the reconstructed thumb, and minimize the risk of recurrent angulation. Although this critique is valid in light of contemporary reconstructive techniques, it does not acknowledge the context in which the Wassel classification was developed. Wassel's manuscript was a retrospective evaluation of the outcomes of surgical ablation, the most common method of treatment at the time, and therefore was not designed in the context of thumb reconstruction procedures; it is not surprising, therefore, that it fails to provide guidance in selecting modern surgical techniques. Nevertheless, Chung et al. [4] recognized this deficiency and proposed an alternative classification based on the anatomic morphologic features of the origin of the duplicated digit to guide surgical decision-making and assess outcomes. Type I (joint type) describes a duplicated digit with its own epiphysis that forms an articulation at its origin from the main digit, which allows for independent motion of the duplicated digit but may show severe translational or angular deformity in the radioulnar plane. Type 2 (single epiphyseal type) indicates thumb duplication in which the accessory and main digits share a common epiphysis (and so share a joint surface), so independent motion of the supernumerary digit is not possible, but translational deformity tends not to be as severe. Type III (osteochondroma-like type) refers to a digit in which the duplicated digit arises from the metaphysis of the main
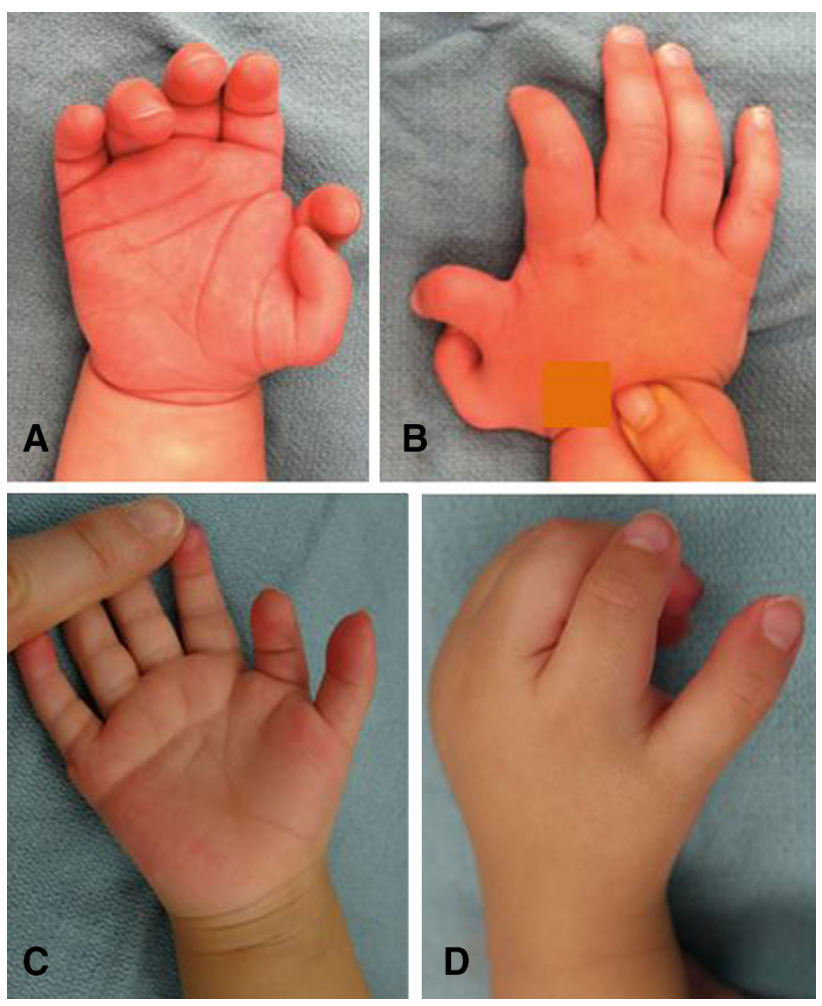

Fig. 3A-D The photographs show the hands of (A) Patient 1 in supination and (B) pronation and $(\mathbf{C})$ for Patient 2 in supination and (D) pronation. Both patients had Type VI thumb duplications. Despite having the same Wassel classification type, the duplications differ regarding the symmetry of thumb development, presence of angular deformity, joint stability, and soft tissue anomalies, which highlights this deficiency of the Wassel classification system. These characteristics are critical to recognize as they inform operative decisionmaking and influence surgical outcomes.

digit, resembling an osteochondroma. Type IV (hypoplastic type) indicates a digit that arises from the dominant digit by soft tissue alone without identifiable osseous connection (Fig. 4). They then described various reconstruction techniques based on their classification involving various combinations of digit excision, ligament and soft tissue reconstruction, corrective osteotomy, and coaptation of skeletal elements from each thumb. Evanson et al. [8] evaluated the prognostic value of the classification of Chung et al. and found a higher reoperation rate in Types I and II compared with Types III and IV.

A final critique of the Wassel classification is that because it is based on radiographic criteria, it may not be possible to characterize all duplicated elements in skeletally immature patients. In particular, it is challenging to determine the presence of physes and epiphyses in younger patients, and thus it may be difficult or impossible to differentiate Type I, III, or V duplication from Type II, IV, or VI, respectively (Fig. 5). Unfortunately, the proposed alternative classification systems also are based on radiographic criteria and therefore are unable to improve on the 


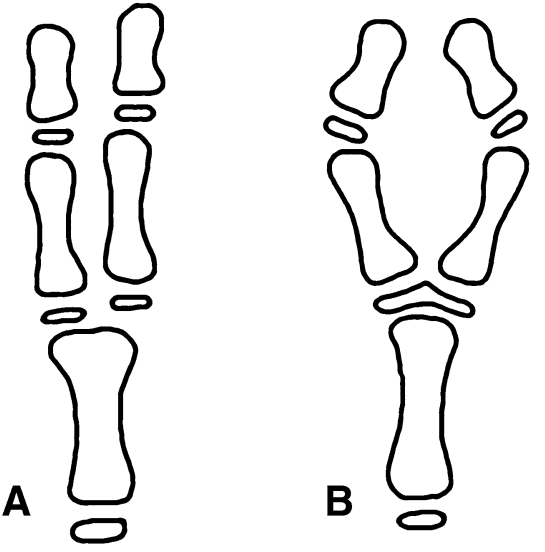

Fig. 4A-D These depictions show the classification of radial polydactyly as described by Chung et al. [4]: (A) Type I is the "joint type," with the origin of the extra digit as an independent articular surface; (B) Type II is the "epiphyseal type," with the origin of the extra digit arising from a shared epiphysis; (C) Type III is the "enchondroma type," with the origin of the extra digit resembling an

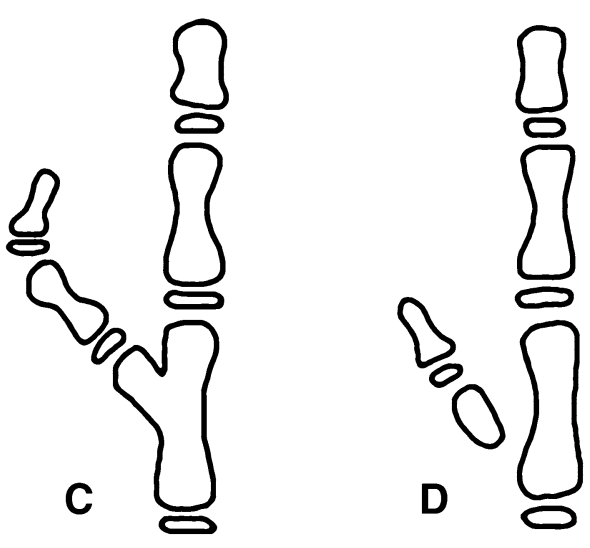

osteochondroma; and (D) Type IV is the "hypoplastic type," with the origin of the extra digit consisting of soft tissue only. (Published with permission from Chung MS, Baek GH, Gong HS, Lee, HJ, Kim J, Rhee SH. Radial polydactyly: proposal for a new classification system based on the 159 duplicated thumbs. J Pediatr Orthop. 2013;33:190_ 196.).
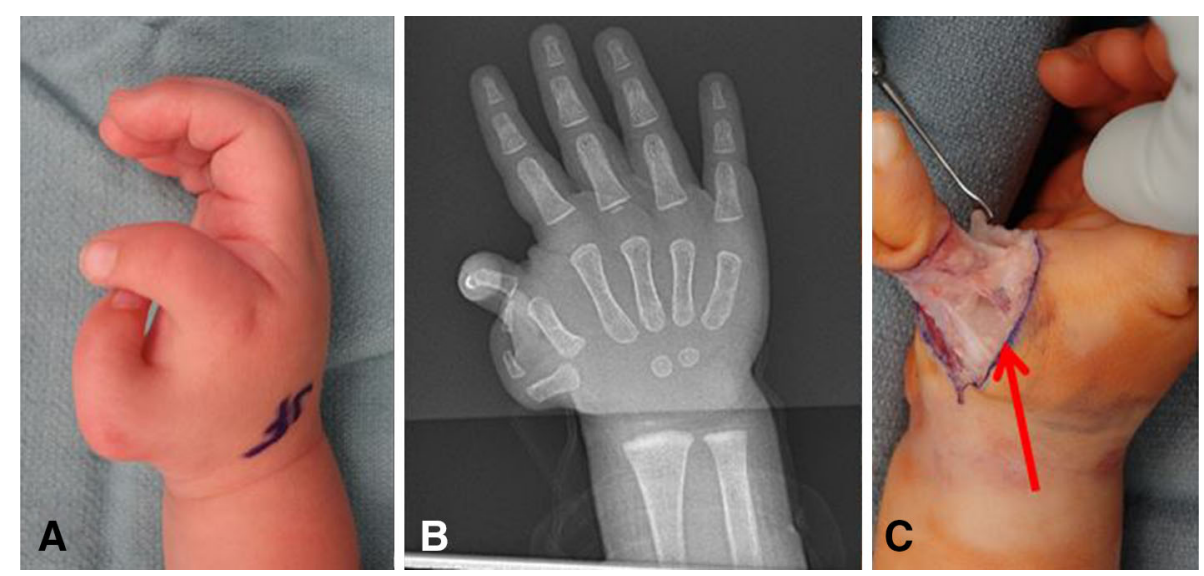

Fig. 5A-C (A) A photograph obtained at the time of the clinical examination, (B) a radiograph, and (C) an intraoperative photograph of a patient with thumb duplication are shown. On clinical examination and on the radiograph, it is not clear whether this represents a Wassel Type V or Type VI thumb duplication. Intraoperatively, a

Wassel classification in this regard. Notably, ossification of the trapezium in Rotterdam Types VII and VIII deformities does not occur until the patient is approximately 5 years old. Similarly, Chung et al. [4] acknowledged the difficulty in differentiating their Type 1 (joint type) from Type 2 (epiphyseal type).

\section{Conclusion}

The Wassel classification of thumb polydactyly, although imperfect, has informed our understanding of the osseous anomalies in radial polydactyly and largely forms the basis cartilaginous bridge between the two metacarpal bases was identified (red arrow), revealing that this is a Type $\mathrm{V}$ thumb duplication and highlighting the limitations of classification systems based on radiographic criteria in skeletally immature patients.

of all subsequent classification systems. Its most important limitation is that it does not account for the anatomic complexity of this congenital hand difference, including soft tissue deficiencies and redundancies, axial plane deformities, joint instability, and functionality. Classification systems, such as the Rotterdam classification, that include designations for complex osseous and soft tissue elements may prove more useful in conveying the full extent of the radial-sided hand deformity and for informing surgical technique; nevertheless, an understanding of the Wassel classification system is important as it serves as the foundation for these subsequent classification systems. Finally, to accurately reflect the genesis of the Wassel 
classification, many pediatric hand surgeons favor referring to this classification system of radial polydactyly as the Flatt classification.

\section{References}

1. Baek GH. Duplication. In: Abzug JM, Kozin SH, Zlotolow DA, eds. The Pediatric Upper Extremity. New York, NY: SpringerVerlag; 2015:325-368.

2. Buck-Gramko D, Behrens P. [Classification of polydactyly of the hand and foot] [in German]. Handchir Mikrochir Plast Chir. 1989;21:195-204.

3. Cabrera Gonzalez M, Perez Lopez LM, Martinez Soto G, Gutierrez de la Iglesia D. Prognostic value of age and Wassel classification in the reconstruction of thumb duplication. $J$ Child Orthop. 2013;7:551-557.

4. Chung MS, Baek GH, Gong HS, Lee, HJ, Kim J, Rhee SH. Radial polydactyly: proposal for a new classification system based on the 159 duplicated thumbs. J Pediatr Orthop. 2013;33:190-196.

5. Dijkman RR, van Nieuwenhoven CA, Selles RW, Habenicht R, Hovius SE. A multicenter comparative study of two classification system for radial polydactyly. Plast Reconst Surg. 2014;134:991-1001.

6. Dijkman RR, van Nieuwenhoven CA, Selles RW, Hovius SE. Comparison of functional outcome scores in radial polydactyly. $J$ Bone Joint Surg Am. 2014; 96:463-470.

7. Egawa T. [Surgical treatment of polydactyly of the thumb][in Japanese]. Keisei Geka. 1966;9:97-105.

8. Evanson BJ. Hosseinzadeh P, Riley SA, Burgess RC. Radial polydactyly and the incidence of reoperation using a new classification system. J Pediatr Orthop. 2016;36:158-160.

9. Ezaki M. Outcome scores: what they do and do not say. Commentary on an article by Robert R. Dijkman, MD, et al.: "Comparison of functional outcome scores in radial polydactyly". J Bone Joint Surg Am. 2014;96:e51.

10. Flatt AE. Extra thumbs. In: Flatt AE, ed. The Care of Congenital Hand Anomalies. St Louis, MO: Quality Medical Publishing; 1977:120-135.

11. Goldfarb CA, Wall LB, Bohn DC, Moen BA, Van Heest AE. Epidemiology of congenital upper limb anomalies in a midwest United States population: an assessment using the Oberg,
Manske, and Tonkin classification. $J$ Hand Surg Am. 2015;40:127-132.

12. Little KJ, Cornwall R. Congenital anomalies of the hand: principles of management. Orthop Clin North Am. 2016;47:153-168.

13. Millesi $H$. [Deformations of the fingers after surgery of polydactylia][in German]. Klin Med Osterr Z Wiss Prakt Med. 1967;22:266-272.

14. Miura T. Triphalangeal thumb. Plast Reconstr Surg. 1976;58: 587-594.

15. Oberg KC, Feenstra JM, Manske PR, Tonkin MA. Developmental biology and classification of congenital anomalies of the hand and upper extremity. J Hand Surg Am. 2010;35:2066-2076.

16. Odiorne JM. Polydactylism in related New England families. $J$ Hered. 1943;34:45-55.

17. Sesgin MZ, Stark RB. The incidence of congenital defects. Plast Reconstr Surg Transplant Bull. 1961;27:261-267.

18. Stutz C, Mills J, Wheeler L, Ezaki M, Oishi S. Long-term outcomes following radial polydactyly reconstruction. J Hand Surg Am. 2014; 39:1549-1552.

19. Swanson AB. A classification for congenital limb malformations. J Hand Surg Am. 1976;1:8-22.

20. Tada K, Yonenobu K, Tsuyuguchi Y, Kawai H, Egawa T. Duplication of the thumb: a retrospective review of two hundred and thirty-seven cases. J Bone Joint Surg Am. 1983;65:584-598.

21. Temtamy SA, McKusick VA. The genetics of hand malformations. Birth Defects Orig Artic Ser. 1978;14:i-xviii, 1-619.

22. Tonkin MA. Thumb duplication: concepts and techniques. Clin Orthop Surg. 2012;4:1-17.

23. Wall LB, Goldfarb CA. Reconstruction for type IV radial polydactyly. J Hand Surg Am. 2015;40:1873-1876.

24. Wassel HD. The results of surgery for polydactyly of the thumb: a review. Clin Orthop Relat Res. 1969;64:175-193.

25. Waters PM, Bae DS. Pediatric Hand and Upper Limb Surgery: A Practical Guide. Philadelphia, PA: Lippincott Williams \& Wilkins; 2012.

26. Watt AJ, Chung KC. Duplication. Hand Clin. 2009;25:215-227.

27. Wenger DR. Book review: Pediatric Hand and Upper Limb Surgery: A Practical Guide. J Pediatr Orthop. 2013;33:468-469.

28. Wood VE. Polydactyly and the triphalangeal thumb. J Hand Surg Am. 1978;3:436-444.

29. Zuidam JM, Selles RW, Ananta M, Runia J, Hovius SE. A classification system of radial polydactyly: inclusion of triphalangeal thumb and triplication. J Hand Surg Am. 2008;33:373-377. 